\title{
Paper Orthogonal Harmonic Signals of the Generalized Class
}

\author{
Vitaliy Balashov, Vasyl Oreshkov, Iryna Barba, and Olena Iegupova \\ O. S. Popov Odessa National Academy of Telecommunications, Odessa, Ukraine
}

https://doi.org/10.26636/jtit.2021.146720

\begin{abstract}
Telecommunications transmission technologies with OFDM rely on orthogonal harmonic signal (OHS) systems. The criteria applicable to synthesizing OHS systems of the generalized class, including both classical signals and signals whose duration exceeds the orthogonality interval, have been considered. The problems of minimizing the effective width of the spectrum of the generalized class OHS have been solved. Estimates of the efficiency of the generalized class OHS have been given.
\end{abstract}

Keywords-Fourier transformation, OFDM, orthogonal harmonic signals, orthogonality interval, signal envelope.

\section{Introduction}

In recent years, thanks to the success of digital signal processing, orthogonal frequency division multiplexing (OFDM) transmission technologies have become increasingly popular in telecommunications and have been relied upon to transmit broadband orthogonal harmonic signal (OHS) systems [1]-[3]. Today, digital subscriber line (xDSL) transmission technologies are, in accordance with recommendations of the International Telecommunication Union (ITU-T) G.992 and G.993, the most common solutions used to offer broadband access via well-developed subscriber networks [4], [5]. In 2014, ITU adopted Recommendation G.9701 defining the characteristics of the G.fast transmission technology. It relies on the OHS and provides signals through a multi-pair telephone cable, with the speed of up to $1 \mathrm{Gbit} / \mathrm{s}$ and in the frequency band of up to $106 \mathrm{MHz}$ [6].

Various OHS systems are used in modern radio access systems, including Wi-Fi (IEEE 802.11), WiMAX (IEEE 802.16), and LTE. They are also relied upon in high-quality digital radio and television broadcasting systems [7]-[10]. The transmission of signals through optical cables is a new area of telecommunications, where OFDM technologies have begun to be used intensively. OHS allows to increase the efficiency of optical transmission technologies relying on dense wavelength division multiplexing (DWDM) [11]-[14].

\section{Problem Statement}

The widespread use of transmission systems (TSs) with OHS in communication networks results from the fact that these technologies ensure high efficiency of transmitting information via communication channels with abnormal and unstable frequencies, as well as with noise characteristics over time.

OHS systems are described by real trigonometric functions which are orthogonal by interval $\tau_{0}$ :

$$
\begin{array}{r}
\left\{\cos \left(l \omega_{0} t\right), \sin \left(l \omega_{0} t\right)\right\}, l=0,1,2, \ldots, N-1, \\
\omega_{0}=2 \pi f_{0}=\frac{2 \pi}{\tau_{0}},-\frac{\tau_{0}}{2} \leq t<\frac{\tau_{0}}{2},
\end{array}
$$

where $N$ is the total number of orthogonal functions in the $0-\omega_{B}$ frequency band, $\omega_{B}=N \cdot \omega_{0}$ is the upper frequency of the transmission channel bandwidth, $\omega_{0}$ is the frequency of the first OHS system harmonic, $\tau_{0}$ is the orthogonality interval.

Examples of orthogonal harmonic signals from Eq. (1) for $l=4,5,6,22$ are given in Fig. 1 .

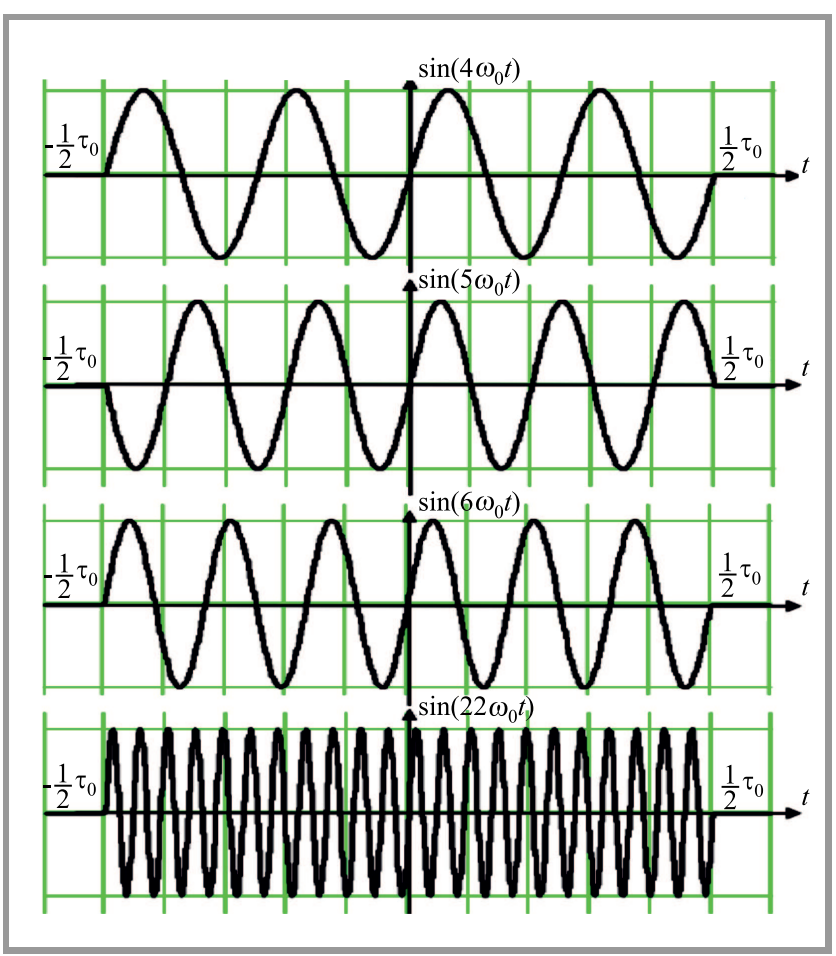

Fig. 1. Orthogonal harmonic signal graphs.

Functions from Eq. (1) are related by the Euler relation:

$$
\mathrm{e}^{\mathrm{i} l \omega_{0} t}=\cos \left(l \omega_{0} t\right)+\mathrm{i} \sin \left(l \omega_{0} t\right)
$$


with exponential time functions:

$$
\left\{\mathrm{e}^{\mathrm{i} l \omega_{0} t}\right\} \begin{gathered}
N-1 \\
l=0
\end{gathered},-\frac{\tau_{0}}{2} \leq t<\frac{\tau_{0}}{2} .
$$

Signal spectra of Eq. (1) have a sufficiently high concentration of energy in the frequency domain (see Fig. 2) and there are effective algorithms for digital processing of OHS systems using Fourier transformation algorithms and providing an acceptable computational complexity of signal modulation-demodulation algorithms in TS with a significant number of signals.

The transformation (spectrum) of Fourier functions given by Eq. (3) is described by the following formula:

$$
\begin{aligned}
S\left(\omega-l \omega_{0}\right)=\int_{-\frac{\tau_{0}}{2}}^{\frac{\tau_{0}}{2}} \mathrm{e}^{\mathrm{i} l \omega_{0} t} \mathrm{e}^{-\mathrm{i} \omega t} \mathrm{~d} t \\
\quad=\frac{\sin \left(\omega-l \omega_{0}\right) \frac{\tau_{0}}{2}}{\left(\omega-l \omega_{0}\right)},-\infty<\omega<\infty .
\end{aligned}
$$

Examples of envelope spectrum graphs defined by Eq. (4) for $l=4,5,6,22$ are shown in Fig. 2 .

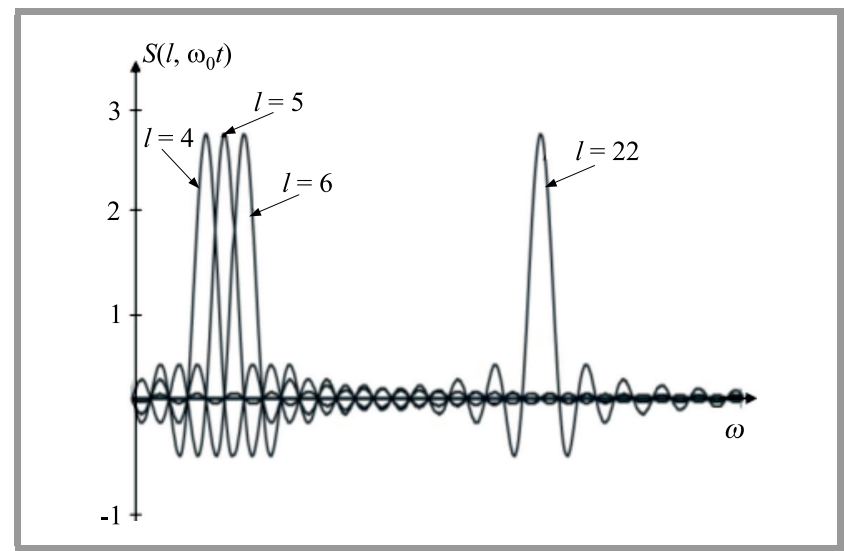

Fig. 2. Example of OHS envelope spectra.

The signal spectra are theoretically infinite and decreases in frequency at a rate proportional to $1 / \omega$. When OHS signals pass through a communication channel with a limited bandwidth, their spectra are truncated. As a result, the orthogonality of the received signals is damaged, and, therefore inter-symbol and inter-channel interference noises are generated. Based on Eq. (1), it is possible to design systems of orthogonal signals with a higher concentration of energy in the frequency domain than with classical OHS. These signals pass through a communication channel with a distorting transmission function. They are less distorted, offer lower interference levels and are more immune to frequency characteristics variations in the communication channel than traditional OHS.

Let us formulate the following OHS synthesis task with energy concentration in the frequency domain that is higher than in the classical OHS, in order to determine the conditions when the system of functions is:

$$
\begin{array}{r}
\left\{\sqrt{u(t)} \mathrm{e}^{\mathrm{i} l \omega_{0} t}\right\}_{l=0}^{N-1},-\frac{T}{2} \leq t<\frac{T}{2}, \\
\omega_{0}=2 \pi f_{0}, \tau_{0}=\frac{1}{f_{0}},
\end{array}
$$

with orthogonal by interval $T$, such as:

$$
\begin{aligned}
& \int_{-\frac{T}{2}}^{\frac{T}{2}} \sqrt{u(t)} \mathrm{e}^{\mathrm{i} l \omega_{0} t} \sqrt{u(t)} \mathrm{e}^{-\mathrm{i} k \omega_{0} t} \mathrm{~d} t \\
&=\left\{\begin{array}{l}
1, l=k, \\
0, l \neq k,
\end{array}, \quad T \geq \tau_{0},\right.
\end{aligned}
$$

where $\sqrt{u(t)}$ is the function describing the envelope of signals and $T$ is the duration of functions.

Signals from Eq. (5) that satisfied Eq. (6) will be referred to OHS of the generalized class.

Based on the Parseval-Plancherel equality and the filtering property of the $\delta$-function, the condition in Eq. (6) can be transformed as:

$$
\begin{array}{r}
\int_{-\frac{T}{2}}^{\frac{T}{2}} \sqrt{u(t)} \mathrm{e}^{\mathrm{i} l \omega_{0} t} \sqrt{u(t)} \mathrm{e}^{-\mathrm{i} k \omega_{0} t} \mathrm{~d} t \\
=\frac{1}{2 \pi} \int_{-\infty}^{\infty} U(\mathrm{i} \omega) 2 \pi \delta\left[\omega-(k-l) \omega_{0}\right] \mathrm{d} \omega \\
=U\left[(k-l) \omega_{0}\right]= \begin{cases}1, & l=k, \\
0, & l \neq k,\end{cases}
\end{array}
$$

where $U(\omega)$ is the Fourier transformation (spectrum) of the function (envelope) $u(t), 2 \pi \delta\left[\omega-(k-l) \omega_{0}\right]$ is the spectrum of exponential signal.

For the discrete frequency function defined by Eq. (7), the Fourier transformation is:

$$
U\left(k \omega_{0}\right)=\int_{-\infty}^{\infty} u(t) \mathrm{e}^{-\mathrm{i} k \omega_{0} t} \mathrm{~d} t=\left\{\begin{array}{ll}
1, & k=1 \\
0, & k \neq 0
\end{array} .\right.
$$

Let us convert the last expression using parameters $T$ and $\tau_{0}$ :

$$
U\left(k \omega_{0}\right)=\sum_{n=-\infty}^{\infty} \int_{-\frac{T}{2}+n \tau_{0}}^{\frac{T}{2}+n \tau_{0}} u\left(t-n \tau_{0}\right) \mathrm{e}^{-\mathrm{i} k \omega_{0} t} \mathrm{~d} t,
$$

and replace the variables $\tau=t-n \tau_{0}$ :

$$
\begin{gathered}
U\left(k \omega_{0}\right)=\sum_{n=-\infty}^{\infty} \int_{-\frac{T}{2}}^{\frac{T}{2}} u(\tau) \mathrm{e}^{-\mathrm{i} k \omega_{0}\left(\tau+n \tau_{0}\right)} \mathrm{d} \tau \\
=\sum_{n=-\infty}^{\infty} \mathrm{e}^{-\mathrm{i} k \omega_{0} n \tau_{0}} \int_{-\frac{T}{2}}^{\frac{T}{2}} u(\tau) \mathrm{e}^{-\mathrm{i} k \omega_{0} \tau} \mathrm{d} \tau .
\end{gathered}
$$

Under the sum sign is the product of the spectrum of the function $u(\tau)$ per exponent. It corresponds to the spectrum of the function delayed by $n \tau_{0}$ :

$$
\begin{aligned}
& U\left(k \omega_{0}\right)=\sum_{n=-\infty}^{\infty} \int_{-\frac{T}{2}}^{\frac{T}{2}} u\left(\tau-n \tau_{0}\right) \mathrm{e}^{-\mathrm{i} k \omega_{0} \tau} \mathrm{d} \tau \\
& =\int_{-\frac{T}{2}}^{\frac{T}{2}} \sum_{n=-\infty}^{\infty} u\left(\tau-n \tau_{0}\right) \mathrm{e}^{-\mathrm{i} k \omega_{0} \tau} \mathrm{d} \tau= \begin{cases}1, & k=0 \\
0, & k \neq 0\end{cases}
\end{aligned}
$$


A function spectrum containing a constant component only corresponds to a function which is constant over time. Therefore:

$$
\sum_{n=-\infty}^{\infty} u\left(\tau-n \tau_{0}\right)=\text { const }
$$

Thus, the following statement has been proved. In order for the signal system from Eq. (5) to be orthogonal, it is necessary for the sum of the shifts of function $u(t)$ (the square of the envelope) by $n \tau_{0}, n= \pm 0, \pm 1, \pm 2, \ldots$ be a constant value (see Fig. 3, where dotted lines are the functions $u(t)$ shifted by $\left.\tau_{0}\right)$.

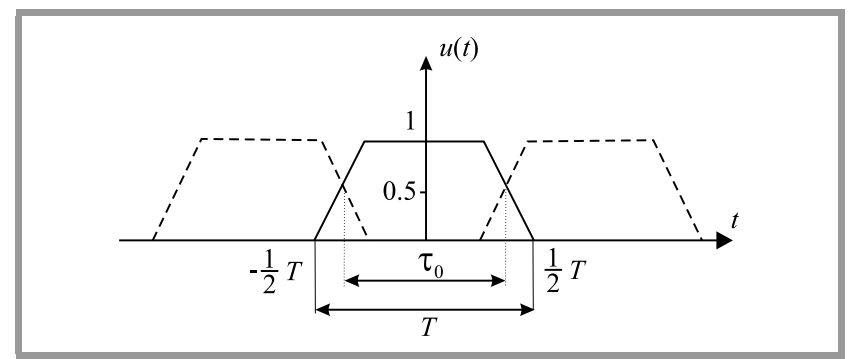

Fig. 3. Graph illustrating criterion.

Note that the condition, defined by Eq. (6), of signal orthogonality from Eq. (5) also describes the rule for correlation reception of OHS signals in the OFDM transmission technology. In this case, it follows from Eq. (6) that the resulting frequency characteristics when receiving signals are determined not by the envelope function $\sqrt{u(t)}$, but by the function $u(t)$ itself. On this basis, further attention will be paid to the synthesis and characteristics of the functions $u(t)$.

\section{Method for Synthesizing OHS Envelope of the Generalized Class}

Here, the method for synthesizing signals $u(t)$, according to the criterion shown in Eq. (12), is presented. Signal $u(t)$ is considered to be the convolution of signal $p(t)=1,-\tau_{0} / 2 \leq t<\tau_{0} / 2$ of a rectangular shape and symmetrical (second order symmetry) in the middle of signal $\varphi(t),-\tau / 2 \leq t<\tau / 2$, where $\tau=T-\tau_{0}$. The resulting signal has edges that are symmetric to the boundaries of the interval $\left(-\tau_{0} / 2, \tau_{0} / 2\right)$, and it meets condition (12).

Signal spectrum $u(t)$ is equal to the product of signal spectra $p(t)-P(\omega)$ and $\varphi(t)-\phi(\omega)$ :

$$
U(\mathrm{i} \omega)=P(\mathrm{i} \omega) \times \phi(\mathrm{i} \omega) .
$$

The following optimization problem defines if $u(t)$ signals synthesized using the proposed method have the minimum effective spectrum width. Obviously, with the same $T$ and $\tau_{0}$ parameters, the spectral characteristics of $u(t)$ signals will be determined by the spectrum of the $\varphi(t)$ signal. Thus, the problem is to find $\varphi(t),-\tau / 2 \leq t<\tau / 2$ with the minimum permissible effective spectrum width.
The formulated rule is a classical optimization problem and has a number of solutions depending on additional optimization conditions [15].

One of the solutions consists in using the pulse described by [15]:

$$
\varphi(t)=\sqrt{\frac{2}{\tau}} \cos \frac{\pi}{\tau} t,-\frac{\tau}{2} \leq t<\frac{\tau}{2} .
$$

A graph of $\varphi(t)$ signal with the minimum effective spectrum is shown in Fig. 4.

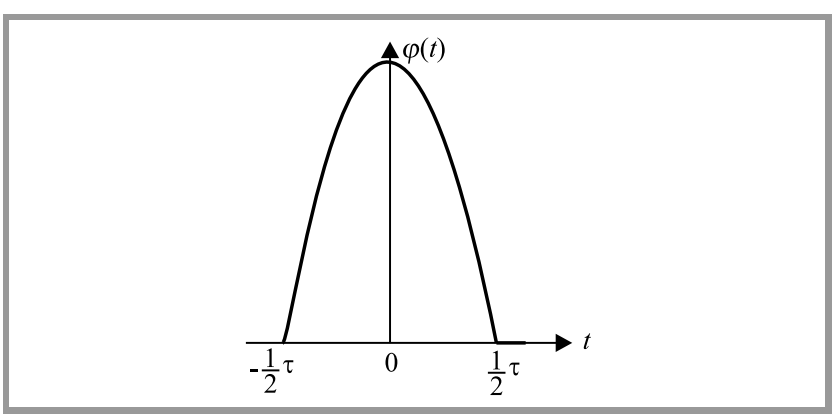

Fig. 4. Pulse of duration $\tau$ with minimum effective spectrum width.

Figure 5 illustrates the given synthesis method of function $u(t)$ in the form of convolution of signal $p(t)=1,-\tau_{0} / 2 \leq$ $t<\tau_{0} / 2$, with signal $\varphi(t),-\tau / 2 \leq t<\tau / 2$, described by the function from Eq. (14).

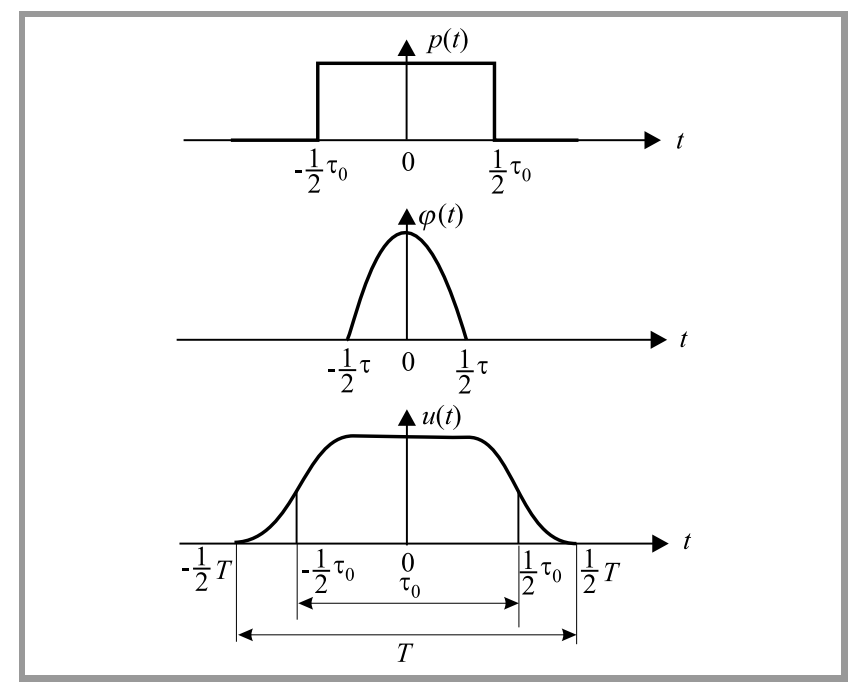

Fig. 5. Convolution of function $\varphi(t)$ with function $p(t)=1$.

Taking into account the corresponding normalization, the optimal function $u(t)$ is described by:

$$
\begin{aligned}
& u(t)= \\
& \begin{cases}1, & \text { at }|t| \leq(1-\alpha) \frac{\tau_{0}}{2} \\
\cos ^{2} \frac{\pi}{4 \alpha}\left[2 f_{0}|t|+\alpha-1\right], & \text { at }(1-\alpha) \frac{\tau_{0}}{2} \leq|t|<(1+\alpha) \frac{\tau_{0}}{2} \\
0, & \text { in all other cases }\end{cases}
\end{aligned}
$$

where $\alpha=\left(T-\tau_{0}\right) / \tau_{0}$ is a signal expansion coefficient. 


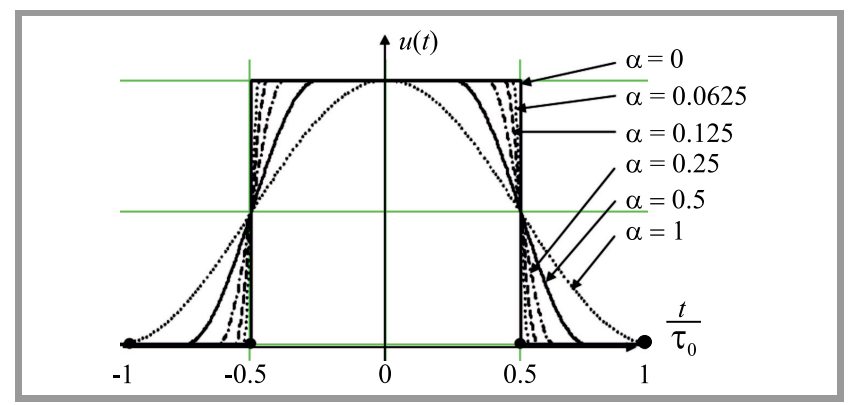

Fig. 6. Optimal $u(t)$ functions.

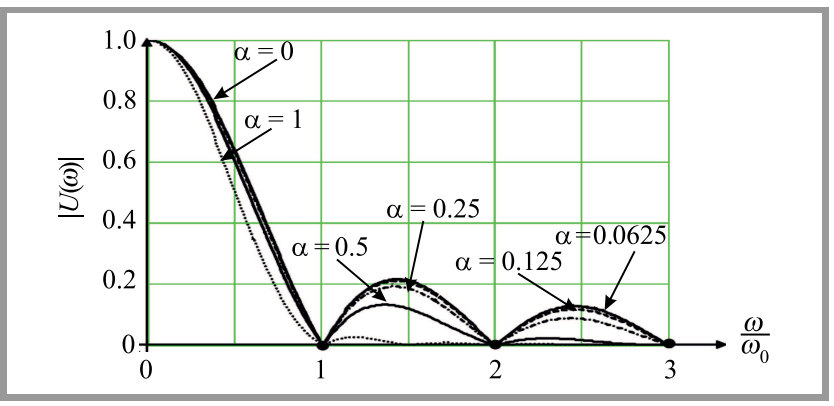

Fig. 7. Spectra module graph $U(\omega)$.

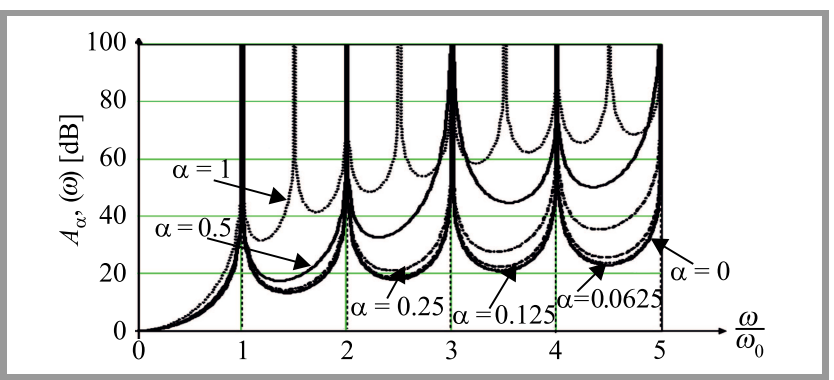

Fig. 8. Graphs of function $A_{\alpha}(\omega)$ of optimal generalized class OHS.

Equation (15) describes a function with cosine quadratic edges. Graph $u(t)$ with signal expansion coefficient values of $\alpha=0,0.0625,0.125,0.25,0.5$ and 1 is given in Fig. 6 . Figure 7 shows the spectra module graphs $U(\omega)$ of optimal functions $u(t)$.

To illustrate the positive energy concentration effect of the spectra generalized class OHS, provided by the optimal functions $u(t)$, let us normalize the energy at frequency $\omega=0$ in decibels:

$$
A_{\alpha}(\omega)=10 \log \frac{U^{2}(0)}{U^{2}(\omega)} .
$$

Graphs $A_{\alpha}(\omega)$ depicting spectral energy concentration calculated by Eq. (16) with signal expansion coefficient values of $\alpha=0,0.0625,0.125,0.25,0.5$ and 1 are shown in Fig. 8. Figure 9 shows examples of orthogonal signals $s_{1}(t)=u(t) \sin \left(\omega_{0} t\right)$ and $s_{2}(t)=u(t) \sin \left(2 \omega_{0} t\right)$ of the generalized class OHS system with functions $u(t)$ being optimal if values of the signal expansion coefficient are $\alpha=0,0.25,0.5$ and 1 .

\section{Extension of the Class of Orthogonal Signals}

The above-described method used for synthesizing generalized class OHS types may be used for synthesis of other OHS systems as well.

It is easy to verify the hypothesis that the temporary function $u_{1}(t),-\tau_{0} \leq t<\tau_{0}$ shown as an example in Fig. 10a, consisting of intervals:

$$
\begin{aligned}
& u_{1-1}(t), \quad-\tau_{0} \leq t<-\frac{7}{8} \tau_{0}, \\
& u_{1-2}(t), \quad-\frac{3}{4} \tau_{0} \leq t<-\frac{1}{2} \tau_{0}, \\
& u_{1-3}(t), \quad-\frac{1}{4} \tau_{0} \leq t<-\frac{1}{8} \tau_{0}, \\
& u_{1-4}(t), \quad \frac{1}{8} \tau_{0} \leq t<\frac{1}{4} \tau_{0}, \\
& u_{1-5}(t), \quad \frac{1}{2} \tau_{0} \leq t<\frac{3}{4} \tau_{0} \text {, and } \\
& u_{1-6}(t), \quad \frac{7}{8} \tau_{0} \leq t<\tau_{0}
\end{aligned}
$$

satisfies condition (12) with displacement if $n \tau_{0}$ for $n= \pm 0, \pm 1, \pm 2$.

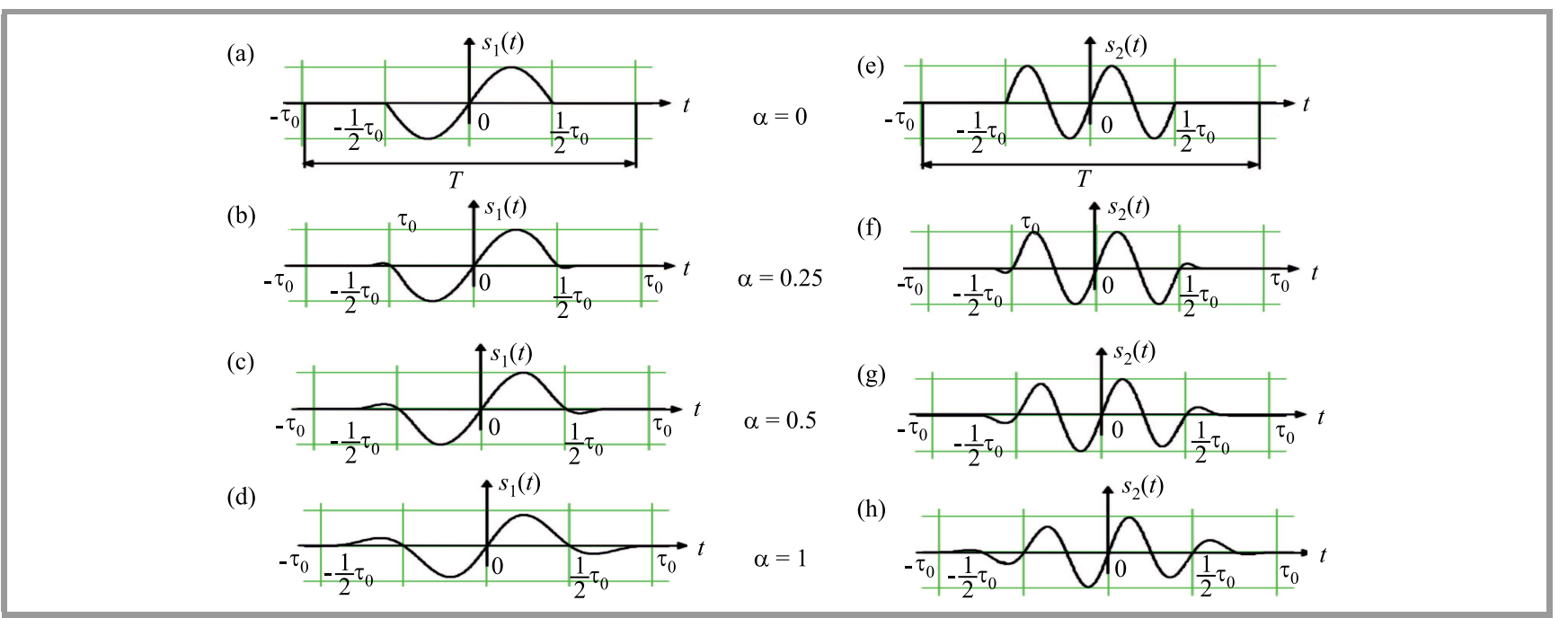

Fig. 9. Examples of the generalized class OHS $s_{1}(t)$ and $s_{2}(t)$ with optimal functions $u(t)$ if $\alpha=0,0.25,0.5$ and 1 . 
Figure 10b shows another function, $u_{2}(t),-\tau_{0} \leq t<\tau_{0}$, also satisfying condition (12). From the graphs of functions $u_{1}(t)$ and $u_{2}(t)$, it is obvious that the given functions are orthogonal.

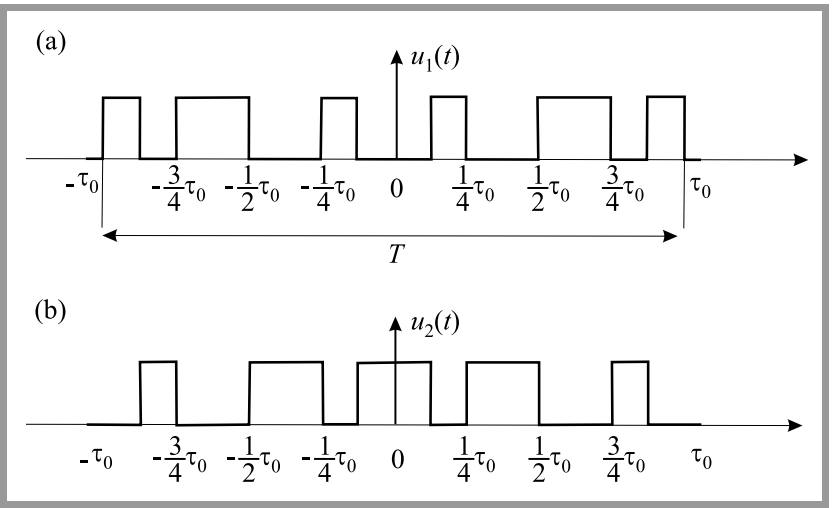

Fig. 10. Example of a piecewise constant functions for the criterion (12).

Hence, it has been justified that the criterion of OHS orthogonality in Eq. (12), at a certain level of $\tau_{0}$ and with the uninterrupted envelopes $u(t)$ considered, also meets the plurality of piecewise-constant functions $u(t)$. Their application allows to synthesize the corresponding OHS systems of the generalized class.

Figure 11 shows examples of the given signals $s_{1}(t)$ and $s_{2}(t)$ of the OHS system with the piecewise constant envelope function $u_{1}(t)$, see Fig. 10a.

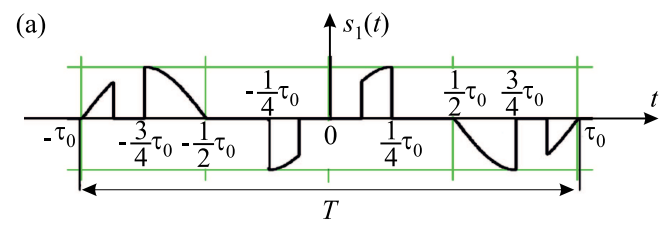

(b)

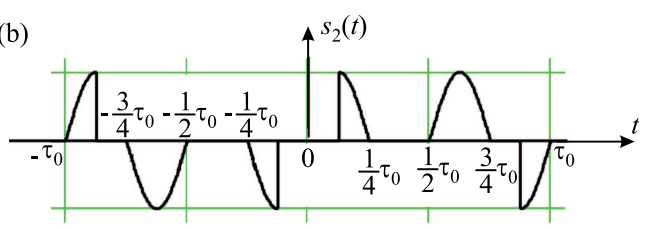

Fig. 11. Examples of OHS $s(t)$ with piecewise constant envelopes with criterion (12).

For the OHS of the generalized class using piecewiseconstant functions $u(t)$, the orthogonality criterion from Eq. (6) should be added with the following conditions:

- signals $u(t)$ are symmetrical along axis $u(0)$,

- interval $\tau_{0}=\sum_{i} \Delta \tau_{i}$, where $\Delta \tau_{i}$ is the duration of $i$-th interval of function $u(t),-\frac{T}{2} \leq t<\frac{T}{2}$,

- the interval of function $u(t)$ satisfies the condition $T \geq 2 \tau_{0}$. (a)

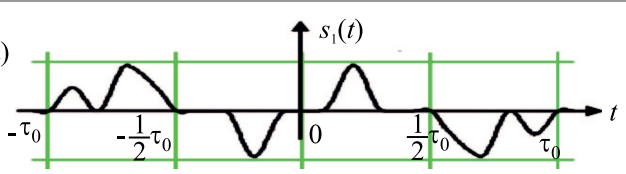

(b)

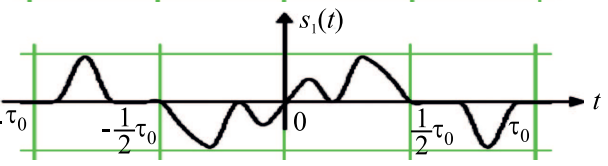

(c)

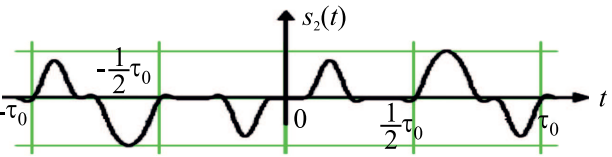

(d)

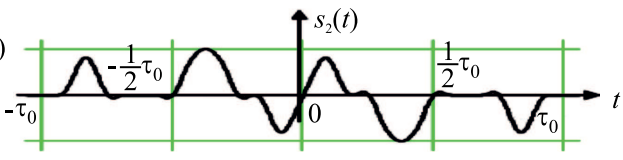

Fig. 12. Examples of OHS $s(t)$ with piecewise constant envelopes with cosine-quadratic fronts $(\alpha=0.0625)$.

It is also easy to verify compliance the criterion (12) of piecewise-constant envelope functions $u(t)$ with fronts having the second-order symmetry. Figure 12 shows examples of such functions $u_{1}(t)$ and $u_{2}(t)$ with cosine-quadratic fronts corresponding to piecewise constant envelope functions $u_{1}(t)$ and $u_{2}(t)$, with the signal expansion coefficient of $\alpha=0.0625$.

\section{Conclusions}

The article defines a generalized class of orthogonal harmonic signals (OHS) and the following results are obtained.

1. The synthesis problem for OHSs that are orthogonal with the interval of $T, \tau_{0} \leq T<2 \tau_{0}$ is presented.

2. Criteria for the synthesis of functions $u(t)$ of the generalized class OHS are defined.

3. Function $u(t)$ is proposed to be synthesized as a convolution of the $p(t)=1,-\frac{1}{2} \tau_{0} \leq t<\frac{1}{2} \tau_{0}$ rectangular signal, symmetrical along the middle of the $\varphi(t),-\frac{1}{2} \tau \leq t<\frac{1}{2} \tau$, signal, where $\tau=T-\tau_{0}$. The edges of the resulting signal are symmetrical relative to the boundaries of the $\left[-\frac{1}{2} \tau_{0}, \frac{1}{2} \tau_{0}\right]$ interval and satisfy condition (12).

4. The problem of optimizing the synthesis for $u(t)$ functions with the minimum effective spectrum width $U(\omega)$ at given $T$ and $\tau_{0}$ parameters is solved. The solution to the problem is a function with cosine-square fronts, known in theory, which is a convincing proof of the correctness (validity) of the authors' proposals presented in the article. 
5. To estimate the energy concentration gain, provided by the optimal $u(t)$ functions, $U(\omega)$ spectra were calculated for different values of the signal expansion coefficient $\alpha$.

6. It has been demonstrated that the OHS orthogonality criterion for a certain selection of $\tau_{0}$, along with the considered $u(t)$ continuous functions, also corresponds to the set of piecewise constant functions $u(t)$, the application of which allows to synthesize the corresponding OHS systems of the generalized class.

\section{References}

[1] V. A. Balashov, P. P. Vorobienko, and L. M. Liakhovetskyi, Systemi Peredachi Ortogonalnimi Garmonicheskimi Signalami. Eko-trendz, 2012 (ISBN: 978-5-88405-096-9) (in Ukrainian).

[2] V. Oreshkov, O. Iegupova, and I. Barba, "Efficiency of generalized class orthogonal harmonic signals application in G.fast transmission systems", in Proc. 14th Int. Conf. on Adv. Trends in Radioelec., Telecommun. and Comp. Engin. TCSET, Slavske, Ukraine, 2018, pp. 987-990 (DOI: 10.1109/TCSET.2018.8336360).

[3] V. Oreshkov, I. Barba, and V. Balashov, "Compensation of crosstalk in the parallel operation of G.fast systems over TPP type multibundle telephone cables", in Proc. Int. Conf. on Inform. and Telecommun. Technol. and Radio Electronics UkrMiCo, Odessa, Ukraine, 2018, pp. 1-5 (DOI: 10.1109/UkrMiCo43733.2018.9047517).

[4] Recommendation ITU-T G.992.5, "Asymmetric digital subscriber line 2 transceivers (ADSL2) - Extended bandwidth ADSL2 (ADSL2plus)" [Online]. Available: https://www.itu.int/rec/ T-REC-G.992.5-200901-I/en

[5] Recommendation ITU-T G.993.2, "Very high speed digital subscriber line transceivers 2 (VDSL2)" [Online]. Available: https://www.itu.int/rec/T-REC-G.993.2-201902-I/en

[6] Recommendation G.9701, "Fast access to subscriber terminals (G.fast) - Physical layer specification" [Online]. Available: https://www.itu.int/rec/T-REC-G.9701-201903-I/en

[7] "Standard for Information Technology - Telecommunications and Information Exchange Between Systems Local and Metropolitan Area Networks - Specific Requirements - Part 11: Wireless LAN Medium Access Control (MAC) and Physical Layer (PHY) Specifications Amendment: Light Communications", IEEE Std 802.11-2016, 2016, pp. 1-3534 (DOI: 10.1109/IEEESTD.2016.7786995).

[8] "Standard for Local and metropolitan area networks Part 16: Air Interface for Broadband Wireless Access Systems", IEEE Std 802.162004, 2019, pp. 1-2080 (DOI: 10.1109/IEEESTD.2009.5062485).

[9] "Radio broadcasting systems; digital audio broadcasting (DAB) to mobile, portable and fixed receivers", ETSI EN 300 401, vol. 2, 2016 [Online]. Available: https://www.etsi.org/deliver/etsi_en/ 300400_300499/300401/02.01.01_20/en_300401v020101a.pdf

[10] "Digital Video Broadcasting (DVB); Framing structure, channel coding and modulation for digital terrestrial television”, ETSI EN 300 744, 2015 [Online]. Available: https://www.etsi.org/deliver/etsi_en/ 300700_300799/300744/01.06.02_60/en_300744v010602p.pdf

[11] D. J. F. Barros and J. M. Kahn, "Optimized dispersion compensation using orthogonal frequency-division multiplexing", J. of Lightwave Technol. vol. 26, no. 16, pp. 2889-2898, 2008 [Online]. Available: https://www.osapublishing.org/jlt/viewmedia.cfm?uri=jlt-26-162889\&seq $=0$
[12] X. Zhang, J. Li, and Z. Li, "SSBI cancellation method for IMDDOFDM system with a single photodiode", in Proc. PIERS, 2014, pp. 2719-2722 (ISBN: 9781510815605).

[13] P. Torres-Ferrera, S. O. Vázquez, and R. Gutiérrez-Castrejón, " $4 \times 100 \mathrm{~Gb} / \mathrm{s}$ WDM DD-OFDM using EAM for next generation Ethernet transceivers over SMF", Optics Commun., vol. 365, pp. 86-92, 2016 (DOI: 10.1016/j.optcom.2015.11.063).

[14] V. A. Vardanyan, "Estimation and compensation of signal-signal beating interference in direct detection fiber-optical transmission systems of OFDM signals", Proc. Optoelectron., Instrument. and Data, vol. 54 , no. 3, 2018, pp. 292-300 (DOI: 3103/S8756699018030123).

[15] R. Kurant and D. Gilbert, Methods of Mathematical Physics. Wiley Eastern Limited, 1953 (ISBN: 9780852261316).

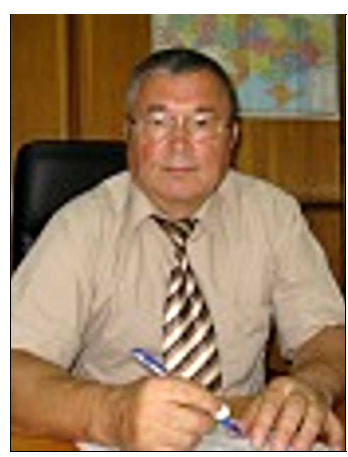

Vitaliy Balashov has a Ph.D. degree in Technical Sciences and is a Professor at the O. S. Popov Odessa National Academy of Telecommunications, Odessa, Ukraine. $\mathrm{He}$ is the author of 170 scientific and educational works, has earned 24 patents for invention, and has published 7 monographs. His research interests include the telecommunication theory and the OFDM system telecommunication theory.

(iD) https://orcid.org/0000-0001-6122-4647

E-mail: bvaoniis@gmail.com

O. S. Popov Odessa National Academy of

Telecommunications

Kuznechnaya, 1,

65029 Odessa, Ukraine

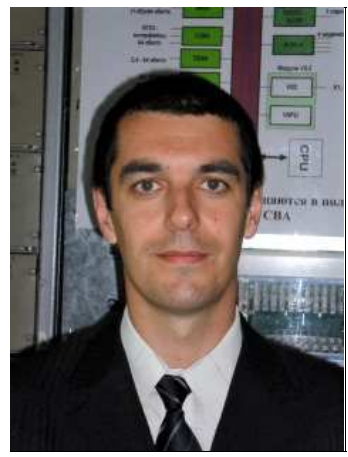

Vasyl Oreshkov holds a Ph.D. in Technical Sciences. $\mathrm{He}$ is a Senior Lecturer at the O. S. Popov Odessa National Academy of Telecommunications, Odessa, Ukraine. He is the author of more than 102 scientific and educational works, including 78 scientific articles and conference abstracts, has earned 2 patents for invention, and has published 7 monographs, as well as 15 educational and methodical manuals. His research interests include the theory of transmission systems with orthogonal harmonic signals, electromagnetic compatibility of transmission systems and development of broadband access networks.

(iD) https://orcid.org/0000-0001-9796-0216

E-mail: Oreshkov_VI@ukr.net

O. S. Popov Odessa National Academy of

Telecommunications

Kuznechnaya, 1,

65029 Odessa, Ukraine 


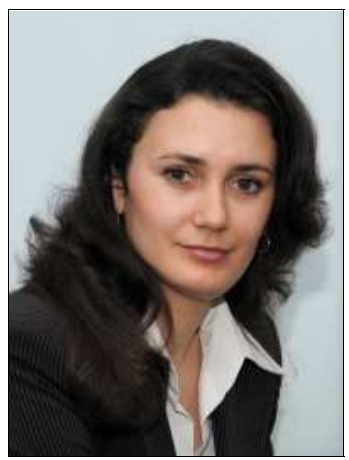

Iryna Barba holds a Ph.D. in Technical Sciences and is a Senior Lecturer at the O.S. Popov Odessa National Academy of Telecommunications, Odessa, Ukraine. She is the author of more than 50 scientific publications, including 30 conference abstracts, 9 of which are indexed in the Scopus scientific-metric database, 18 articles, 1 patent and 2 monographs.

(D) https://orcid.org/0000-0002-6751-0086

E-mail: ibarba@onat.edu.ua

O. S. Popov Odessa National Academy of

Telecommunications

Kuznechnaya 1,

65029 Odessa, Ukraine

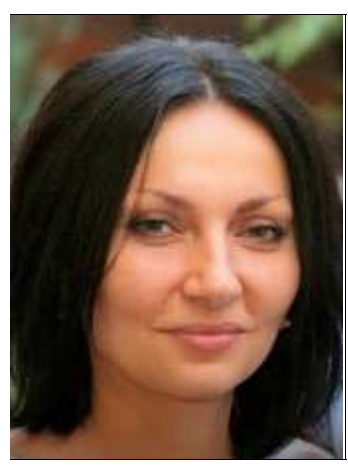

Olena Iegupova is a graduate student of the O.S. Popov

Odessa National Academy of

Telecommunications, Odessa,

Ukraine. She is the author of 18 scientific and educational works, including 5 scientific articles, 12 conference abstracts and 1 monograph. Her research interests include improving the efficiency of broadband signal transmission technologies over telephone networks.

(iD) https://orcid.org/0000-0003-1540-2988

E-mail: elena.iegupova@gmail.com

O. S. Popov Odessa National Academy of Telecommunications

Kuznechnaya, 1,

65029 Odessa, Ukraine 